Taxonomía y sistemática

\title{
Un nuevo registro para Lepanthes (Orchidaceae) de México y clarificación del estatus taxonómico de Lepanthes quetzalensis
}

\section{A new report of Lepanthes (Orchidaceae) for México and clarification of the taxonomic status of Lepanthes quetzalensis}

\author{
Derio Antonio Jiménez-López ${ }^{\mathrm{a}, *}$, Rodolfo Solano ${ }^{\mathrm{b}}$, Rubén Martínez-Camilo ${ }^{\mathrm{a}}$ \\ y Nayely Martínez-Meléndez ${ }^{\mathrm{a}}$ \\ a Instituto de Ciencias Biológicas, Universidad de Ciencias y Artes de Chiapas, Libramiento Norte Poniente Núm. 1150 Colonia Lajas Maciel, 29039 Tuxtla \\ Gutiérrez, Chiapas, México \\ ${ }^{\mathrm{b}}$ Centro Interdisciplinario de Investigación para el Desarrollo Integral Regional Unidad Oaxaca, Instituto Politécnico Nacional, Hornos 1003, Colonia \\ Nochebuena, 71230 Santa Cruz Xoxocotlán, Oaxaca, México
}

Recibido el 14 de enero de 2017; aceptado el 25 de junio de 2017

Disponible en Internet el 7 de diciembre de 2017

\begin{abstract}
Resumen
El género neotropical Lepanthes es uno de los grupos más grandes dentro de Orchidaceae. Aquí se reporta por primera vez la presencia de Lepanthes fratercula en la flora de México. Además, se clarifica el estatus taxonómico de L. quetzalensis, considerándola como una especie diferente de L. scopula, en la cual había sido incluida como su sinónimo. Tanto L. fratercula como L. quetzalensis son descritas a partir de ejemplares mexicanos recolectados en el estado de Chiapas. Para cada taxón se proporciona información sobre su distribución, hábitat, fenología y estatus de conservación en México; adicionalmente, ambas especies son comparadas con otros taxones morfológicamente similares de América Central. La riqueza de Lepanthes para la flora mexicana se incrementa a 65 especies, siendo 35 de ellas nativas de Chiapas.
\end{abstract}

(C) 2017 Universidad Nacional Autónoma de México, Instituto de Biología. Este es un artículo Open Access bajo la licencia CC BY-NC-ND (http://creativecommons.org/licenses/by-nc-nd/4.0/).

Palabras clave: Chiapas; Lephantes fratercula; Lephantes scopula; Pleurothallidinae; Reserva de la Biosfera El Triunfo

\section{Abstract}

The Neotropical genus Lepanthes is one of the largest groups within Orchidaceae. Here the presence of Lepanthes fratercula is reported by the first time for the Mexican flora. In addition, the taxonomic status of L. quetzalensis is clarified here, considering it as a different specie from L. scopula, in which it has been included as its synonym. Both $L$. fratercula and L. quetzalensis are described from Mexican specimens collected in Chiapas State. For each taxon information about its distribution, habitat, phenology, and conservation status in Mexico is provided. Additionally, both species are compared with other morphologically similar taxa from Central America. The richness of Lepanthes in the Mexican flora increases to 65 species, 35 of which are native to Chiapas.

(C) 2017 Universidad Nacional Autónoma de México, Instituto de Biología. This is an open access article under the CC BY-NC-ND license (http://creativecommons.org/licenses/by-nc-nd/4.0/).

Keywords: Chiapas; Lephantes fratercula; Lephantes scopula; Pleurothallidinae; El Triunfo Biosphere Reserve

\footnotetext{
* Autor para correspondencia.

Correo electrónico: derio.a@ hotmail.com (D.A. Jiménez-López).

La revisión por pares es responsabilidad de la Universidad Nacional Autónoma de México.
} 


\section{Introducción}

Lepanthes $\mathrm{Sw}$. es uno de los géneros de mayor riqueza específica de la familia Orchidaceae, con una estimación de 1,120 especies (Chase et al., 2015; Karremans, 2016). La cifra actual se incrementa cada año debido a la descripción de nuevas especies (p. ej. Damian y Larsen, 2017). La distribución geográfica del género es neotropical, desde el Eje Volcánico Transversal en México, hasta Bolivia y el norte de Brasil, incluyendo las Antillas; el grupo alcanza su mayor riqueza en las regiones andinas de Colombia y Ecuador (Betancur, Sarmiento, ToroGonzález y Valencia, 2015; Luer y Thoerle, 2012; Pridgeon, Cribb, Chase y Rasmussen, 2005). El tratamiento monográfico para Lepanthes de México realizado por Salazar y Soto (1996) reportó la presencia de 60 especies en el país. Pero unos años después fueron adicionadas 3 especies para México: Lepanthes maldonadoae Soto Arenas (Soto y Solano, 2003a), L. mazatlanensis Solano et Reynaud (Solano, 2003) y L. yunckeri Ames ex Yuncker (Soto y Solano, 2003b). Así, la riqueza del género se incrementó a 63 especies, de las cuales 43 son endémicas al país.

Durante un estudio en desarrollo para determinar la distribución altitudinal de epífitas vasculares en la Reserva de la Biósfera El Triunfo, en el estado de Chiapas, se recolectaron ejemplares de Orchidaceae pertenecientes al género Lepanthes. Cuando el material fue examinado para su determinación, comparándolo con las especies del género reportadas para México y Guatemala en la literatura especializada (Archila, 2001, 2002; Catling y Salazar, 1994; Luer, 1990, 1991; Salazar y Soto, 1996; Solano, 2003; Soto, 2003; Soto y Solano, 2003a, b), se encontró que correspondían a taxones que no habían sido registrados en la flora mexicana, o al menos no con un nombre considerado con estatus correcto: Lepanthes fratercula Luer et Béhar y Lepanthes quetzalensis Luer et Béhar. La región de Chiapas donde fueron recolectados estos ejemplares, el Soconusco, ha sido relativamente bien explorada botánicamente desde hace 8 décadas, especialmente para epífitas vasculares (Damon, Almeida-Cerino, Valle-Mora, Bertolini y López-Urbina, 2015; Long y Heath, 1991; Martínez-Meléndez, Pérez-Farrera y Farrera-Sarmiento, 2008; Martínez-Meléndez, Pérez-Farrera y Martínez-Camilo, 2009; Martínez-Meléndez, Martínez-Camilo, Pérez-Farrera y Martínez-Meléndez, 2011; Martínez-Camilo, Pérez-Farrera y Martínez-Meléndez, 2012; Matuda, 1950a, b; Pérez-Farrera, 2004; Pérez-Farrera y Miceli-Méndez, 2004; Pérez-Farrera, Martínez-Camilo, Martínez-Meléndez, FarreraSarmiento y Maza-Villalobos, 2012; Solano et al., 2016). No obstante, el trabajo florístico ahí realizado en los últimos años sigue proporcionando novedades para la flora de México (Solano, Jiménez-Machorro y Damon, 2011; Solano, Salazar y Jiménez-Machorro, 2011; Solano, Damon, Cruz-Lustre, Jiménez-Bautista, Avendaño-Vázquez.y Bertolini, 2016).

Lepanthes fratercula fue descrita en 1990 a partir de ejemplares recolectados en los departamentos de Baja Verapaz y Jalapa, Guatemala (Luer, 1990). Posteriormente, Mó-Mó y Ruiz-Cruz (2012) descubrieron otra localidad guatemalteca en Alta Verapaz y recientemente fue registrada también en el occidente de Honduras, en el departamento de Lempira (Vega, Cetzal-Ix, Mó-Mó y Romero-Soler, 2016). En la descripción original de la especie los autores mencionaron que es muy similar a L. guatemalensis Schltr., de la cual se distingue por el menor tamaño de sus plantas y flores. Sin embargo, otras especies del género descritas de Guatemala son más similares a L. fratercula, como L. enca-barcenae Archila, L. luisii Archila, L. necae Archila, L. stenosepala Luer et Béhar y L. uvallensis Archila.

Lepanthes quetzalensis también fue descrita en 1990 a partir de ejemplares de Guatemala recolectados en los departamentos de Alta y Baja Verapaz (Luer, 1990). Mó-Mó y Ruíz-Cruz (2012) descubrieron posteriormente una población adicional en Alta Verapaz, por lo que se consideraba como un endemismo guatemalteco. En la descripción original de L. quetzalensis los autores no compararon esta especie con alguno de sus congéneres de la región morfológicamente similares, como L. scopula Schltr. y L. turialvae Rchb. f. Posteriormente, Salazar y Soto (1996), Dix y Dix (2000) y Soto (2003) señalaron a L. quetzalensis como sinónimo de L. scopula. Estas últimas especies constituyen un complejo que se reconoce por presentar hojas ovadas, a veces acuminadas en el ápice, inflorescencia pectinada más corta que la hoja, con pedicelos muy aproximados entre sí, por lo general recargada sobre el envés de la hoja, flores amarillas, a veces manchadas de rojizo y los pétalos bilobados más largos que las láminas del labelo, con los lóbulos generalmente diferentes en forma y tamaño. Otras especies de Centroamérica integrantes de este complejo son L. atrata Endres ex Luer, L. erubescens Bogarín, Pupulin et Karremans, L. isabelae Archila, L. kleinii Bogarín et Pupulin, L. pristidis Rchb. f., L. samacensis Ames, L. turialvae Rchb. f. y L. velifera Luer et Béhar. El reconocimiento de la mayoría de ellas como especies ha sido posible solo en las últimas 3 décadas (Archila, 2001, 2002; Bogarín, Karremans y Pupulin, 2012; Luer, 1990, 1991; Pupulin y Bogarín, 2012); en el pasado probablemente fueron confundidas con L. scopula o L. turialvae (Ames y Correll, 1952; Hamer, 1981; McLeish, Pearce, Adams y Briggs, 1995).

En este trabajo se da a conocer el primer reporte de Lepanthes fratercula para la flora mexicana y se reconoce con estatus taxonómico específico a L. quetzalensis, no como un sinónimo de L. scopula. Se presentan descripciones y fotos de L. fratercula y L. quetzalensis basadas en ejemplares mexicanos, así como un mapa que muestra sus localidades conocidas. Además se presenta una ilustración de L. quetzalensis para ayudar a clarificar su estatus taxonómico.

\section{Materiales y métodos}

Las descripciones de L. fratercula y L. quetzalensis y la ilustración de esta última se basaron en el análisis de material vegetal recolectado en la Reserva de la Biosfera El Triunfo y el Parque Nacional Lagunas de Montebello, ambos en Chiapas, México, así como en fotografías tomadas en el hábitat de cada especie. No se presenta la ilustración para L. fratercula debido a que su estatus taxonómico es claro. Se prepararon ejemplares de respaldo que fueron depositados en el Herbario HEM de la Universidad de Ciencias y Artes de Chiapas. La ilustración se realizó usando un microscopio estereoscópico y un tubo de extensión; la 
ilustración incluye el hábito de la planta, vista de la flor, disección floral y vistas detalladas de pétalos, labelo y columna.

Para verificar la correcta determinación de las especies se consultó literatura especializada para el género Lepanthes en Mesoamérica (Archila, 2001, 2002; Bogarín et al., 2012; Luer, 1990, 1991; Pupulin y Bogarín, 2012; Salazar y Soto, 1996; Solano, 2003; Soto, 2003; Soto y Solano, 2003a, b), así como la consulta de información disponible en línea en Epidendra (http://www.epidendra.org), Tropicos (http://www.tropicos.org) y de los herbarios AMO, HEM, MEXU, OAX y MO (Thiers, 2017).

Las localidades de los ejemplares hasta ahora conocidos de L. fratercula y L. quetzalensis fueron georreferenciadas y, con ayuda de ArcGIS 10.2 (ESRI, 2012), fueron sobrepuestas en un mapa que incluyó la parte sureste de México y noroeste de Centroamérica.

\section{Redescripciones}

Lepanthes fratercula Luer et Béhar, Lindleyana 5(3): 188. 1990

Tipo. Guatemala. Baja Verapaz: epiphytic in forest near Unión Barrios, montaña El Quisis, aldea El Carmen, 1,800 m, 15 Jan. 1989, M. Béhar 9 (holotipo en MO!).

Hierba epífita, erecta, cespitosa, hasta $5.5 \mathrm{~cm}$ de alto incluyendo la inflorescencia. Raíces delgadas, flexuosas, blanquecinas, hasta $18 \mathrm{~mm}$ de largo. Tallos erectos, cilíndricos, formados por hasta 5 entrenudos, envueltos totalmente por vainas lepanthiformes, 7-20 mm largo, $2 \mathrm{~mm}$ diámetro. Hojas coriáceas, elípticas, redondeadas, 2-lobuladas y cortamente mucronadas en el ápice, 9-12 x 5-6 mm, abruptamente atenuadas en un peciolo canaliculado, de $1 \mathrm{~mm}$ de largo. Inflorescencia más larga que la hoja, racemosa, recargada sobre la superficie abaxial de la hoja, 15-25 mm de largo; pedúnculo filiforme, recto, tan largo como la hoja, $10-12 \mathrm{~mm}$ de largo; raquis flexuoso, hasta con 6 flores que abren en sucesión, progresivamente alargándose con el desarrollo de nuevas flores. Bráctea floral oblicuamente infundibuliforme, obtusa, $1 \mathrm{~mm}$ de largo. Flores no resupinadas, más o menos extendidas en la antesis, de 10 x $3.4 \mathrm{~mm}$; sépalos amarillo claro, el dorsal esfumado de rojo, pétalos, labelo y antera de color púrpura. Ovario obcónico, $1 \mathrm{~mm}$ de largo, articulado a un pedicelo cilíndrico y recto, de $1.5 \mathrm{~mm}$ de largo. Sépalo dorsal cóncavo, ovado, agudo, cortamente caudado, ligeramente revoluto en los márgenes, 3-nervado, 4 x 2 mm; sépalos laterales unidos entre sí ca. 2/5 de su longitud, ligeramente convexos, triangular-lanceolados, ligeramente oblicuos, acuminado-caudados, 3-nervados, 4.5 x $1 \mathrm{~mm}$. Pétalos transversalmente bilobulados, diminutamente emarginados, microscópicamente pubescentes, 1-nervados, $0.25 \times 1.2 \mathrm{~mm}$; lóbulo superior oblicuamente ovado, agudo; lóbulo inferior más largo que el superior, angostamente oblongo-lanceolado. Labelo 2-laminado, fusionado a la columna arriba de su base; láminas envolviendo a la columna, de $1.2 \mathrm{~mm}$ de largo, arqueadas, oblongo-lanceoladas, redondeadas en ambos extremos, ensanchadas hacia el extremo apical, microscópicamente pubescente. Columna delgada, recta, $1 \mathrm{~mm}$ de largo. Antera dorsal, ovada, truncada hacia el ápice y la base. Polinios 2, unidos por una gota de viscidio. Estigma apical. Cápsula no vista. (figs. 1A-B).

Espécimen examinado. México. Chiapas: municipio Ángel Albino Corzo, Reserva de la Biosfera El Triunfo, sendero El Triunfo, aproximadamente $300 \mathrm{~m}$ al noroeste del campamento. $15^{\circ} 39^{\prime} 05^{\prime \prime} \mathrm{N}, 92^{\circ} 48^{\prime} 53^{\prime}$ O O 2, 114 m” bosque mesófilo de montaña, 20 diciembre 2014, D. A. Jiménez-López y J. Navarro-Ramos 103 (HEM!).

Distribución. México (Chiapas), Guatemala (Alta Verapaz, Baja Verapaz y Jalapa) y Honduras (Lempira). La población mexicana de L. fratercula fue descubierta en la Sierra Madre de Chiapas, la cual resulta ser la más occidental en la distribución de la especie (fig. 2A).

Hábitat. La especie es una epífita del bosque mesófilo de montaña. Se ha encontrado sobre los tallos de Amphitecna montana L.O. Williams (Bignoniaceae),

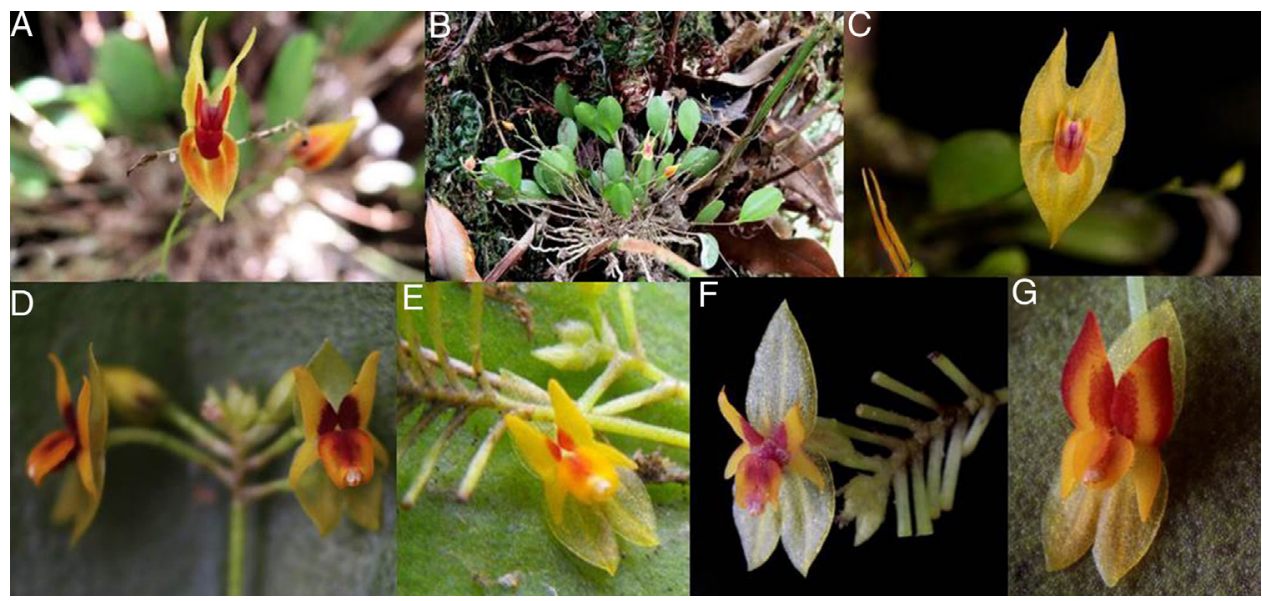

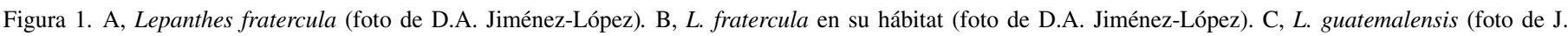

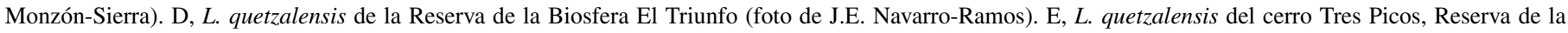

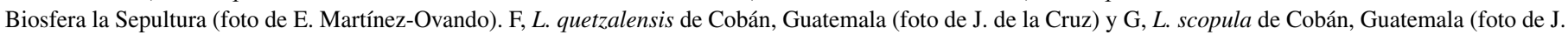
de la Cruz). 

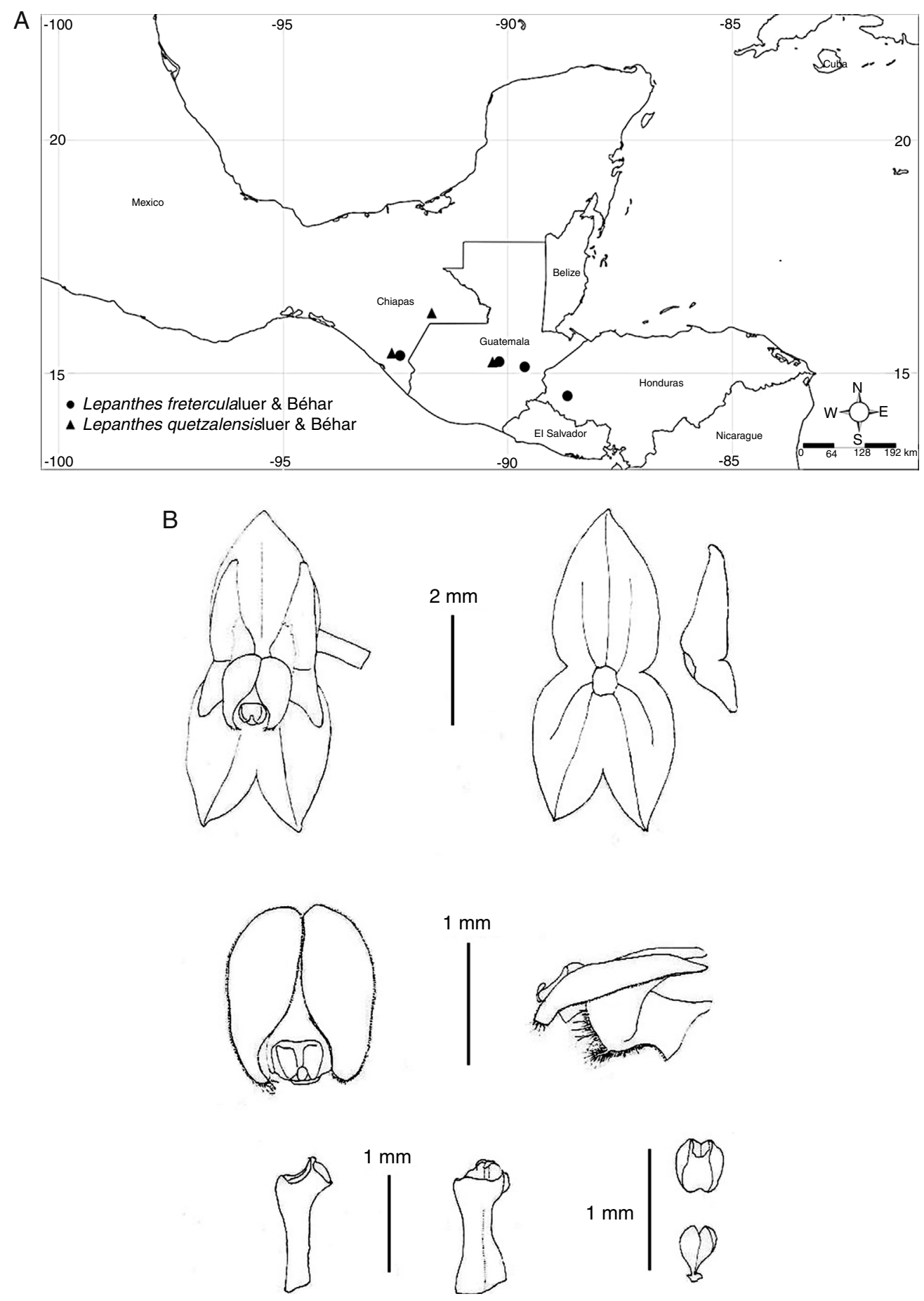

Figura 2. A, mapa de las localidades conocidas de Lepanthes fratercula (círculo negro) y L. quetzalensis (triángulo negro). B, ilustración de Lepanthes quetzalensis Luer et Béhar. Basado en M. Soto s.n., (modificado del dibujo publicado por Salazar y Soto [1996; fig. 64], reproducido con permiso del Instituto Chinoín A.C.).

Chionanthus ligustrinus (Sw.) Pers. (Oleaceae), Matudaea trinervia Lundell (Hamamelidaceae) y Ternstroemia lineata DC. (Pentaphylacaceae).

Fenología. En su hábitat la floración ha sido registrada en diciembre.

Comentarios. La especie es similar y cercana a Lepanthes guatemalensis Schltr., sin embargo, L. fratercula se distingue porque la flor es casi el doble de longitud respecto a $L$. guatemalensis (Luer, 1990; figs. 1A-C), además L. fratercula es muy similar en su morfología floral a varias especies que han sido descritas de Guatemala, entre ellas L. luisii Archila, L. encabarcenae Archila, L. necae Archila, L. stenosepala Luer et Béhar y $L$. uvallensis Archila. La especie más similar parece ser $L$. stenosepala, la cual se distingue por el pedúnculo de la inflorescencia al menos 2 veces más largo que la hoja (vs. ligeramente más largo que la hoja), flores de mayor tamaño y resupinadas (vs. no resupinadas), sépalos rojizos y concoloros (vs. amarillos y el dorsal esfumado de rojo) y el lóbulo superior de los 
pétalos oblongo y obtuso (vs. triangular y agudo). Lepanthes luissi se distingue de L. fratercula por sus sépalos rojizos y concoloros (vs. amarillos y el dorsal esfumado de rojo), sépalos laterales agudos y convergentes hacia los ápices (vs. acuminados y divergentes hacia los ápices) y láminas del labelo oblongas (vs. oblanceoladas). Lepanthes necae difiere de L. fratercula por el seno inconspicuo que se forma en la unión del sépalo dorsal con los laterales (vs. seno conspicuo), el sépalo dorsal triangularovado (vs. ovado), los sépalos laterales subparalelos entre sí (vs. divergentes) y las láminas del labelo oblicuamente ovadas (vs. oblanceoladas). Lepanthes enca-barcenae se distingue de L. fratercula por sus sépalos rojizos, concoloros (vs. amarillos y el dorsal esfumado de rojo), sépalos laterales subparalelos entre sí y agudos en el ápice (vs. divergentes y acuminado-caudados en el ápice) y los lóbulos de los pétalos subiguales en forma y tamaño (vs. diferentes en forma y tamaño). Finalmente, L. uvallensis es diferente de L. fratercula por el seno inconspicuo que se forma en la unión del sépalo dorsal con los laterales (vs. conspicuo), sépalo dorsal ovado-lanceolado no caudado (vs. ovado y cortamente caudado), sépalos laterales subparalelos entre sí y agudos en el ápice (vs. divergentes y acuminado-caudados en el ápice).

Estatus de conservación. No evaluado. La única localidad mexicana conocida de la especie se encuentra en la zona núcleo de la Reserva de la Biosfera El Triunfo, lo cual podría garantizar la viabilidad de la población y la protección de su hábitat. No obstante, el hábitat puede ser vulnerable a los efectos relacionados con el cambio climático, los cuales son mayores cuando se combinan con efectos debido al mal manejo de las zonas agrícolas adyacentes a la reserva (Soto, Solano y Hágsater, 2007).

Lepanthes quetzalensis Luer et Béhar, Lindleyana 5(3): 194. 1990

Tipo. Guatemala. Baja Verapaz: epiphytic in mountain forest near the Quetzal Preserve between Cobán and Unión Barrios, 1,600 m, 11 Feb. 1990, M. Béhar, J. Luer et C.A. Luer 7 (holotipo en $\mathrm{MO}$ !).

Hierba epífita, erecta, cespitosa, hasta $4.5 \mathrm{~cm}$ de alto incluyendo la inflorescencia. Raíces delgadas, flexuosas, blanquecinas, hasta $32 \mathrm{~mm}$ de largo. Tallos erectos, cilíndricos, formados por hasta 3 entrenudos, envueltos totalmente por vainas lepanthiformes, $12 \mathrm{~mm}$ de largo, $2 \mathrm{~mm}$ diámetro. Hojas coriáceas, verdosas, ligeramente cóncavas, elípticas, obtusa, 2lobuladas y diminutamente mucronadas en el ápice, marginadas, $2.8 \times 2.3 \mathrm{~cm}$, atenuadas en un peciolo canaliculado, de $3.1 \mathrm{~mm}$ de largo. Inflorescencia casi de la mitad del largo de la hoja, racemosa, recargada sobre la superficie abaxial de la hoja, 15-25 mm de largo; pedúnculo filiforme, recto, con 2 brácteas cortamente tubulares, oblicuas y agudo-acuminadas en el ápice, $2 \mathrm{~mm}$ de largo; raquis pectinado, con los pedicelos dísticos y agrupados entre sí, hasta con 7-8 flores, 1-2 abriendo simultáneamente. Bráctea floral oblicuamente infundibuliforme, obtusa, ligeramente carinada y apiculada, $2 \mathrm{~mm}$ de largo. Flores resupinadas, extendidas en la antesis, ca. $6 \times 2.5 \mathrm{~mm}$; sépalos amarillo traslúcidos, pétalos amarillos, con una mancha púrpura en la base del margen interior del lóbulo superior, labelo amarillo-naranja y esfumado de púrpura hacia la base y márgenes de las láminas, columna púrpura, antera blanquecina y esfumada de púrpura. Ovario obcónico, arqueado, algo ensanchado hacia el ápice, $2 \mathrm{~mm}$ de largo, articulado a un pedicelo cilíndrico y recto, de $0.9 \mathrm{~mm}$ de largo. Sépalo dorsal ligeramente cóncavo, ovado, obtuso, cortamente apiculado y revoluto en el ápice, 3-nervado, ca. $3 \times 2 \mathrm{~mm}$; sépalos laterales unidos entre sí ca. $2 / 3$ de su longitud, ligeramente cóncavos, oblicuamente ovados, obtusos y ligeramente recurvados en el ápice, ligeramente divergentes en el extremo apical, 2-nervados, ca. 2.7 x $1.3 \mathrm{~mm}$. Pétalos transversalmente bilobulados, emarginados, microscópicamente pubescentes, 1-nervados, 1 x $2.5 \mathrm{~mm}$; lóbulo superior oblicuamente triangular-lanceolado, agudo, ligeramente incurvado en el ápice, recto a lo largo de los márgenes, ca. 1 × 2 mm; lóbulo inferior mucho más corto que el superior, oblicuamente triangular, agudo, ligeramente incurvado en el ápice, recto a lo largo del margen externo, cóncavo en el margen interno, ca. 1 x $0.5 \mathrm{~mm}$. Labelo 2-laminado, fusionado casi a la mitad de la columna; las láminas oblicuamente ovadas, redondeadas en el extremo proximal, agudas y dirigidas hacia el interior en el extremo distal, casi rectas a lo largo del margen externo, en contacto entre sí en la base y ocultando la columna excepto por su ápice, de 1.5 x $0.6 \mathrm{~mm}$, diminutamente ciliados. Columna delgada, recta, $1.4 \mathrm{~mm}$ de largo, ligeramente ensanchada hacia el ápice, no sobrepasando la altura de las láminas del labelo. Antera dorsal, ovada, truncada hacia el ápice y la base, apicalmente emarginada, ca. $0.5 \mathrm{~mm}$ de largo. Polinios 2, clavados, unidos por una gota de viscidio. Estigma apical, en forma de cavidad hemielíptica. Cápsula no vista (figs. 1D-F y fig. 2B).

Especímenes examinados. México. Chiapas. Municipio Jaltenango, Reserva El Triunfo, Polígono 1, Cerro El Triunfo, $15^{\circ} 39^{\prime} \mathrm{N}, 92^{\circ} 48^{\prime} \mathrm{O}, 2,100 \mathrm{~m}$, bosque mesófilo de montaña, 21 Nov. 1990, M. Heath y A. Long 1311A (MEXU!); municipio Villa Flores, Cerro Tres Picos, $2.5 \mathrm{~km}$ al SO de Nueva Independencia, $16^{\circ} 11^{\prime} 53.43^{\prime \prime} \mathrm{N}, 93^{\circ} 36^{\prime} 03.24^{\prime \prime} \mathrm{O}, 1,995 \mathrm{~m}$, bosque mesófilo de montaña con Podocarpus matudae, 22 May. 2010, E. Martínez s.n. (OAX[foto]!), Reserva de la Biosfera la Sepultura (fig. 1E); municipio Ángel Albino Corzo, Reserva de la Biosfera El Triunfo, sendero El Triunfo, aprox. $300 \mathrm{~m}$ al NO del campamento, $15^{\circ} 39^{\prime} 05^{\prime \prime} \mathrm{N}, 92^{\circ} 48^{\prime} 53^{\prime \prime} \mathrm{O}, 2,114 \mathrm{~m}$, bosque mesófilo de montaña, 20 Dic. 2014, D. A. Jiménez-López y J. Navarro-Ramos 105 (HEM!); municipio La Trinitaria, Parque Nacional Lagunas de Montebello, bosque mesófilo de montaña, M. Soto s.n. (AMO!).

Distribución. México (Chiapas) y Guatemala (Alta Verapaz y Baja Verapaz). En México la especie se ha registrado en las regiones de la meseta central y sierra Madre de Chiapas (fig. 2A).

Hábitat. La especie es un epífita en los fragmentos más húmedos del bosque mesófilo de montaña, se ha encontrado sobre tallos de Podocarpus matudae Lundell, entre 1,400 y 2,114 m de elevación. Para la Reserva de la Biosfera el Triunfo, Las plantas crecen sobre tallos y ramas de árboles de Amphitecna montana, Chionanthus ligustrinus, Glossostipula concinna (Standl.) Lorence (Rubiaceae), Matudaea trinervia, Symplococarpon flavifolium Lundell (Pentaphylacaceae) y Ternstroemia lineata.

Fenología. En su hábitat la floración ha sido registrada de noviembre a diciembre y en mayo. 
Comentarios. Entre las especies del género distribuidas en América Central, L. quetzalensis es muy similar, tanto en el hábito de las plantas como en la morfología floral, a L. atrata, L. erubescens, L. isabelae, L. kleinii, L. pristidis, L, samacensis, L. turialvae y L. velifera. De entre todas ellas, la especie más similar a L. quetzalensis es L. scopula (figs. 1D-G), de México (Oaxaca y Chiapas), Guatemala y Belice, con la cual puede ser simpátrica, pero esta se distingue por sus sépalos laterales evidentemente apiculados y divergentes en el ápice (vs. diminutamente apiculados y divergentes), pétalos proporcionalmente más grandes con relación al tamaño de la flor, sobrepuestos entre sí por encima de la columna, amarillos con una banda roja a lo largo del margen interno y externo (vs. no sobrepuesto encima de la columna, amarillos con una mancha roja en la base del margen interno del lóbulo superior), el lóbulo superior del pétalo oblicuamente ovado y obtuso (vs. oblicuamente triangular y agudo), el inferior extendiéndose más allá del ápice de la columna (vs. terminando antes o a nivel del ápice de la columna) y las láminas del labelo conspicuamente pubescentes (vs. glabras o glandulosas). Lepanthes pristidis, la otra especie del complejo presente en México, es diferente de L. quetzalensis por sus hojas de color cobrizo, acuminadas en el ápice (vs. verdosas y obtusas en el ápice), pétalos amarillos con bandas de color rojizo en los márgenes externo e interno, papilosos (vs. amarillos con una mancha rojiza en la base del margen interno del lóbulo superior, glabros) y lóbulos superiores de los pétalos sobrepuestos entre sí, oblicuamente ovados y ampliamente obtusos (vs. bien separados entre sí, oblicuamente triangulares y acuminados).

Lepanthes atrata, hasta ahora solo conocida de Costa Rica, se distingue de L. quetzalensis por el sépalo dorsal agudo (vs. obtuso), pétalos amarillos con una banda rojiza a lo largo del margen interno (vs. amarillos con una mancha rojiza en la base del margen interno del lóbulo superior), lóbulo superior de los pétalos oblicuamente ovado y redondeado (vs. triangular-lanceolado y agudo) y láminas del labelo elípticas (vs. oblicuamente ovadas). Lepanthes erubescens, hasta ahora solo registrada en Costa Rica, se distingue de L. quetzalensis por su hábito péndulo, inflorescencia recargada sobre la superficie abaxial de la hoja, sépalos laterales unidos entre sí cerca de la base (vs. unidos cerca del ápice), pétalos proporcionalmente grandes en relación al tamaño de las flores y ligeramente sobrepuestos por encima de la columna (vs. completamente separados por encima de la columna) y láminas del labelo ovado-lanceoladas (vs. oblicuamente ovadas). Lepanthes isabelae, aparentemente un endemismo de Guatemala, es diferente de L. quetzalensis por su inflorescencia no pectinada, sépalo dorsal proporcionalmente más ancho, los sépalos laterales unidos entre sí casi hasta la mitad de su longitud (vs. unidos entre sí más allá de la mitad de su longitud), lóbulos de los pétalos subiguales en forma y tamaño (vs. lóbulo superior de mayor tamaño y forma diferente al inferior) y láminas del labelo oblanceoladas (vs. oblicuamente ovadas). Lepanthes kleinii, hasta ahora solo conocido de la localidad tipo en Costa Rica, se distingue de L. quetzalensis por su hábito péndulo (vs. erecto), sépalo dorsal ovado-lanceolado y agudo (vs. ovado y obtuso), pétalos amarillos con bandas de color rojizo en los márgenes externo e interno (vs. amarillos con una mancha rojiza en la base del margen interno del lóbulo superior), lóbulos de los pétalos subiguales en forma (vs. diferentes en forma), láminas del labelo elípticas (vs. oblicuamente ovadas) y columna extendiéndose más allá de las láminas del labelo (vs. a la misma altura de las láminas del labelo). Lepanthes samacencis, conocida de México, Guatemala, El Salvador y Honduras, es diferente de L. fratercula por sus flores no resupinadas (vs. resupinadas), sépalos laterales no divergentes en los ápices (vs. ligeramente divergentes), pétalos rojo oscuro y amarillo hacia el margen externo, ligeramente sobreponiéndose por encima de la columna (vs. amarillos con una mancha rojiza en la base del margen interno del lóbulo superior y no sobrepuestos entre sí por encima de la columna), con los lóbulos subiguales (vs. lóbulos diferentes en forma y tamaño) y láminas del labelo lineal-lanceoladas (vs. oblicuamente ovadas). Lepanthes velifera Luer et Béhar, hasta ahora solo conocida de Guatemala, se distingue de L. fratercula por sus hojas atenuadoacuminadas en el ápice (vs. obtusas), inflorescencia recargada sobre la superficie adaxial de la hoja (vs. sobre la superficie abaxial), flores no resupinadas (vs. resupinadas), sépalos laterales unidos entre sí cerca de la mitad (vs. unidos hasta cerca del ápice), pétalos cortamente apiculados (vs. no apiculados) y láminas del labelo densamente pubescentes en la mitad apical (vs. glabras).

Lepanthes turialvae fue descrita de Costa Rica y en el pasado ha sido reportada para toda América Central y México, sobre todo porque bajo este nombre se incluyó a L. scopula como su sinónimo (Ames y Correll, 1952; McLeish et al., 1995). Sin embargo, Dix y Dix (2000) y Soto y Salazar (1996) consideraron que ambos nombres correspondían a especies diferentes y que los ejemplares de México y Guatemala determinados como L. turialvae en realidad corresponden a L. scopula. Las ilustraciones y fotos publicadas por Hamer (1981) y McLeish et al. (1995), las cuales fueron determinadas como L. turialvae, corresponden más bien a L. scopula. Así, L. turialvae podría estar restringida solo a Costa Rica o extender su distribución a zonas adyacentes de Nicaragua y Panamá. Esta especie se distingue de L. quetzalensis por sus flores no resupinadas (vs. resupinadas), sépalos amarillos, los laterales rojos a lo largo de la nervadura media (vs. amarillos y concoloros), pétalos proporcionalmente grandes en relación al tamaño de la flor, con lóbulos oblicuamente ovados (vs. triangulares) y láminas del labelo elípticas u ovado-elípticas (vs. oblicuamente ovadas).

Estatus de conservación. Las poblaciones mexicanas de L. quetzalensis se localizan dentro de áreas naturales protegidas, las reservas de la biósfera El Triunfo y La Sepultura, así como el Parque Nacional Lagunas de Montebello; así, la protección de las poblaciones y su hábitat podrían estar garantizados. Sin embargo, la vegetación del Parque Nacional Lagunas de Montebello fue severamente afectada en el pasado reciente por prácticas agrícolas y ganaderas, asentamientos humanos, actividades de turismo no controlado y el mal manejo de fuego en los terrenos agrícolas. No se conocen los impactos que estos factores tuvieron sobre la biodiversidad en este parque, pero se ha sugerido que pudieron provocar la extinción local de algunas especies de orquídeas con alta fidelidad por su hábitat y que en México solo se conocían de esta región (Soto et al., 2007). 


\section{Comentarios taxonómicos}

Con el descubrimiento en Chiapas de L. fratercula y el reconocimiento de $L$. quetzalensis como especie diferente de $L$. scopula, la riqueza para este género en México se incrementa a 65 especies; 43 de ellas son endemismos del país, mientras que 35 son nativas de Chiapas. La presencia de las poblaciones mexicanas de L. fratercula y L. quetzalensis amplía la distribución conocida de ambas especies, las cuales hasta hace poco eran consideradas endemismos restringidos de Guatemala; la primera de ellas, recientemente ha sido registrada también en Honduras. Destaca la importancia del Soconusco, Chiapas, una región de alta biodiversidad y bien explorada botánicamente, en la cual aún es posible descubrir novedades para la flora orquideológica mexicana; además de L. fratercula, en la década actual ahí se han descubierto para el país Acianthera herrerae (Luer) Solano et Soto Arenas, Maxillaria brunnea Linden et Rchb. f., Oncidium poikilostalix (Kränzl.) M.W. Chase et N.H. Williams, Stelis annedamoniae Solano, S. hagsaterii Solano, S. soconuscana Solano y Telipogon helleri (L.O. Williams) N.H. Williams et Dressler (Damon et al., 2015; Solano, Jiménez-Machorro et al., 2011; Solano, Salazar et al., 2011; Solano et al., 2016).

\section{Agradecimientos}

A Juan Gálvez y Jorge Navarro Ramos por su apoyo durante el trabajo de campo. A Edgar Mó y Gerardo Salazar por su ayuda en la corroboración taxonómica. A Miguel A. Pérez-Farrera por las facilidades para trabajar en el herbario HEM. A Roberto García Martínez por la ayuda en la medición de estructuras florales. E. Martínez-Ovando, José Monzón Sierra y Jorge de la Cruz proporcionaron material fotográfico, además a los últimos 2 por facilitar información sobre las localidades en Guatemala.

\section{Referencias}

Ames, O. y Correll, D. S. (1952). Orchids of Guatemala. Fieldiana: Botany, 26, $1-395$.

Archila, F. L. (2001). Lepanthes de Guatemala: monografía del Género Lepanthes Sw. (Orchidaceae) para Guatemala. Guatemala, Guatemala: Editorial Kamar.

Archila, F. L. (2002). Nuevas especies para la flora orquideológica guatemalteca. Guatemalensis, 5, 1-24.

Betancur, J., Sarmiento, H., Toro-González, L. y Valencia, J. (2015). Plan para el estudio y la conservación de las orquídeas en Colombia. Bogotá: Textos: Ministerio de Ambiente y Desarrollo Sostenible, Colombia; Universidad Nacional de Colombia.

Bogarín, D., Karremans, A. P. y Pupulin, F. (2012). Three new Lepanthes (Orchidaceae: Pleurothallidinae) from South-east Costa Rica. Lankesteriana, 12, 107-114.

Catling, P. M. y Salazar, G. A. (1994). Taxonomic notes on Lepanthes disticha (Orchidaceae: Pleurothellidinae) and its allies in Mexico and Northern Central America. Acta Botanica Mexicana, 28, 41-55.

Chase, M. W., Cameron, K., Freudenstein, J., Pridgeon, A. M., Salazar, G., van den Berg, C., et al. (2015). An updated classification of Orchidaceae. Botanical Journal of the Linnean Society, 177, 151-174.

Damian, A. y Larsen, B. (2017). Three new species of Lepanthes (Pleurothalldinae: Orchidaceae) from Amazonas, Peru. Phytotaxa, 291, 149-156.

Damon, A., Almeida-Cerino, C., Valle-Mora, J., Bertolini, V. y López-Urbina, J. H. (2015). Ravines as refuges for Orchidaceae in Southeastern México. Botanical Journal of the Linnean Society, 178, 283-297.
Dix, M. A. y Dix, M. W. (2000). Orchids of Guatemala- a revised annotated checklist. Monographs in Systematic Botany from Missouri Botanical Garden, 78, 1-61.

ESRI. (2012). ArcGIS Desktop, Release 10. Evironmental Systems Research Institute.

Hamer, F. (1981). Las orquídeas de El Salvador III. Sarasota, Florida: The Mary Selby Botanical Gardens.

Karremans, A. P. (2016). Genera Pleurothallidinarum: an updated phylogenetic overview of Pleurothallidinae. Lankesteriana, 16, 219-241.

Long, A. y Heath, M. (1991). Flora of the El Triunfo Biosphere Reserve, Chiapas, Mexico: a preliminary floristic inventory and the plant communities of Polygon 1. Anales del Instituto de Biología, Universidad Nacional Autónoma de México, Serie Botánica, 75, 133-172.

Luer, C. A. (1990). New species of Lepanthes from Guatemala. Lindleyana, 5, $182-198$

Luer, C. A. (1991). New species of Lepanthes (Orchidaceae). Lindleyana, 6, 64-83.

Luer, C. A. y Thoerle, L. (2012). Icones Pleurothallidinarum XXXII. Lepanthes of Colombia (Orchidaceae). Monographs in Systematic Botany from the Missouri Botanical Gardens, 123, 1-296.

Martínez-Camilo, R., Pérez-Farrera, M. A. y Martínez-Meléndez, N. (2012). Listado de plantas endémicas y en riesgo de la Reserva de la Biosfera El Triunfo, Chiapas, México. Botanical Sciences, 90, 263-285.

Martínez-Meléndez, N., Martínez-Camilo, R., Pérez-Farrera, M. A. y MartínezMeléndez, J. (2011). Las epífitas de la Reserva El Triunfo, Chiapas. Tuxtla Gutiérrez, Chiapas: Colección Jaguar, Universidad de Ciencias y Artes de Chiapas.

Martínez-Meléndez, J., Pérez-Farrera, M. A. y Farrera-Sarmiento, O. (2008). Inventario florístico del cerro El Cebú y zonas adyacentes en la Reserva de la Biosfera El Triunfo (Polígono V), Chiapas, México. Boletín de la Sociedad Botánica Mexicana, 82, 21-40.

Martínez-Meléndez, N., Pérez-Farrera, M. A. y Martínez-Camilo, R. (2009). The vascular epiphyte flora of the El Triunfo Biosphere Reserve, Chiapas, México. Rhodora, 111, 503-535.

Matuda, E. (1950a). A contribution to our knowledge of the wild flora of Mt. Ovando, Chiapas. The American Midland Naturalist, 43, 195-223.

Matuda, E. (1950b). A contribution to our knowledge of the wild and cultivated flora of Chiapas. I. Districts Soconusco and Mariscal. The American Midland Naturalist, 44, 513-616.

McLeish, I., Pearce, N. R., Adams, B. R. y Briggs, J. S. (1995). Native orchids of Belize. Rotterdam, Holanda: A.A. Balkema.

Mó-Mó, E. A. y Ruiz-Cruz, E. A. (2012). Estudio de la orquideoflora de la reserva privada Chicacnab, Alta Verapaz, Guatemala. Lankesteriana, 12, 175-190.

Pérez-Farrera, M. A. (2004). Flora y vegetación de la Reserva de la Biosfera el Triunfo: diversidad riqueza y endemismo. En M. A. Pérez-Farrera, N. Martínez-Meléndez, A. Hernández-Yáñez, y A. Arreola-Muñoz (Eds.), La Reserva de la Biosfera El Triunfo: tras una década de conservación (pp. 77-100). Tuxtla Gutiérrez, Chiapas: Universidad de Ciencias y Artes de Chiapas.

Pérez-Farrera, M. A., Martínez-Camilo, R., Martínez-Meléndez, N., FarreraSarmiento, O. y Maza-Villalobos, S. (2012). Listado florístico del cerro Quetzal (Polígono III) de la Reserva de la Biosfera El Triunfo, Chiapas, México. Botanical Sciences, 90, 1-30.

Pérez-Farrera, M. A. y Miceli-Méndez, C. (2004). Epifitas de la Reserva de la Biosfera el Triunfo: composición e importancia. En M. A. Pérez-Farrera, N. Martínez-Meléndez, A. Hernández-Yáñez, y A. Arreola-Muñoz (Eds.), La Reserva de la Biosfera El Triunfo: tras una década de conservación (pp. 101-116). Tuxtla Gutiérrez, Chiapas: Universidad de Ciencias y Artes de Chiapas.

Pridgeon, A. M., Cribb, P. J., Chase, M. W. y Rasmussen, F. N. (2005). Genera Orchidacearum. Vol. 4 Epidendroideae (Part One). Oxford: Oxford University Press.

Pupulin, F. y Bogarín, D. (2012). Lepanthes novae tapatiensis. Orchid Digest, 76, 20-29.

Salazar, G. A. y Soto, M. A. (1996). El género Lepanthes Sw. en México. México D.F.: Asociación Mexicana de Orquideología, A.C. 
Solano, R. (2003). Lepanthes mazatlanensis Solano et Reynaud. En E. Hágsater y M. Soto (Eds.), Icones Orchidacearum 5 and 6, Orchids of Mexico part 2 and 3. México D.F.: Instituto Chinoín A.C.

Solano, R., Damon, A., Cruz-Lustre, G., Jiménez-Bautista, L., AvendañoVázquez, S., Bertolini, V., et al. (2016). Diversity and distribution of the orchids of the Tacana-Boqueron region, Chiapas, Mexico. Botanical Sciences, 94, 625-656.

Solano, R., Jiménez-Machorro, R. y Damon, A. A. (2011). Two new records and one rediscovery for Orchidaceae of México. Acta Botanica Mexicana, 96, 59-72.

Solano, R., Salazar, G. A. y Jiménez-Machorro, R. (2011). New combinations in Orchidaceae of Mexico. Acta Botanica Mexicana, 97, 49-56.

Soto, M. A. (2003). Lepanthes scopula Schltr. En E. Hágsater y M. A. SotoArenas (Eds.), Icones Orchidacearum 5 and 6, Orchids of Mexico part 2 and 3. México D.F.: Instituto Chinoín A.C.
Soto, M. A. y Solano, R. (2003a). Lepanthes maldonadoae Soto Arenas. En E. Hágsater y M. A. Soto-Arenas (Eds.), Icones Orchidacearum 5 and 6, Orchids of Mexico part 2 and 3. D.F., México: Instituto Chinoín A.C.

Soto, M. A. y Solano, R. (2003b). Lepanthes yunckerii Ames ex Yuncker. En E. Hágsater y M. A. Soto-Arenas (Eds.), Icones Orchidacearum 5 and 6, Orchids of Mexico part 2 and 3. México D.F.: Instituto Chinoín A.C.

Soto, M. A., Solano, R. y Hágsater, E. (2007). Risk of extinction and patterns of diversity loss in Mexican orchids. Lankesteriana, 7, $114-121$.

Thiers, B. (2017). Index Herbariorum: a global directory of public herbaria and associated staff. New York Botanical Garden's Virtual Herbarium.. Recuperado el 22 de junio de 2017, de: ht tp://sweetgum.nybg.org/science/ih/

Vega, H., Cetzal-Ix, W., Mó-Mó, E. y Romero-Soler, K. (2016). Nuevos registros para la flora de Honduras y el Parque Nacional Montaña de Celaque. Acta Biológica Colombiana, 21, 635-644. 